\title{
The Asian Option Pricing when Discrete Dividends Follow a Markov-Modulated Model
}

\author{
Yingyi Fang1, Huisheng Shu ${ }^{1 *}$, Xiu Kan ${ }^{2 *}$, Xin Zhang ${ }^{1}$, Zhiwei Zheng1 \\ ${ }^{1}$ School of Science, Donghua University, Shanghai, China \\ ${ }^{2}$ School of Electronic and Electrical Engineering, Shanghai University of Engineering Science, Shanghai, China \\ Email: *hsshu@dhu.edu.cn, *xiu.kan@sues.edu.cn
}

How to cite this paper: Fang, Y.Y., Shu, H.S., Kan, X., Zhang, X. and Zheng, Z.W. (2017) The Asian Option Pricing when Discrete Dividends Follow a Markov-Modulated Model. Open Journal of Statistics, 7, 1067-1080

https://doi.org/10.4236/ojs.2017.76074

Received: October 27, 2017

Accepted: December 26, 2017

Published: December 29, 2017

Copyright $\odot 2017$ by authors and Scientific Research Publishing Inc. This work is licensed under the Creative Commons Attribution International License (CC BY 4.0).

http://creativecommons.org/licenses/by/4.0/

\begin{abstract}
This paper is concerned with the pricing problem of the discrete arithmetic average Asian call option while the discrete dividends follow geometric Brownian motion. The volatility of the dividends model depends on the Markov-Modulated process. The binomial tree method, in which a more accurate factor has been used, is applied to solve the corresponding pricing problem. Finally, a numerical example with simulations is presented to demonstrate the effectiveness of the proposed method.
\end{abstract}

\section{Keywords}

Arithmetic Average Asian Call Option, Discrete Dividends, Geometric Brownian Motion, Markov-Modulated Volatility, Binomial Tree

\section{Introduction}

The option is a contract that gives the owner a right to purchase or sell a certain amount of asset (the underlying asset) at the agreed price (the strike price) within the prescribed time limit, see [1] [2] [3]. It is a financial instrument based on the futures, which gives the owner the right without the obligation. Asian options are path-dependent securities whose payoffs depend on the average of the underlying asset price during the life of the option, see [4] [5] [6] [7]. The financial operators are interested with such options since it could reduce the risk of the volatility inherent in the option and the market mani-pulation of the underlying asset near the expiry dates. Asian options can be divided into geometric mean Asian options and arithmetic average Asian options. Form the solution of arithmetic average Asian options does not exist, so we need to use the numerical approximation approach to calculate the option price. There are 
various kind of numerical methods have been used to calculate the option pricing such as binomial tree method, Monte Carlo method and finite difference method for solving Black-Scholes partial differential equations, etc., see [8] [9] [10] [11].

Kemma and Vorst [12] solved the pricing problem of the discrete arithmetic average Asian option by using Monte Carlo method. Hull and White [13] priced the path-dependent option under the framework of the binomial tree by introducing the state variables. Rogers and Shi [14] calculated the Asian options with finite difference method. Hishida and Yasutomi [15] revealed the relationship between European Options and Asian Options, and obtained the approximate price of the Asian option based on the relationship. Boyle and Potapchik [16] provided a summary of the different methods of pricing Asian options and gave the approaches for computing price sensitivities, the methods discussed include Monte Carlo simulation, finite difference approach and various quasi analytical approaches and approximations.

The use of binomial tree model in option pricing has been very popular since the appearance of the pioneering work by Cox, et al. [17] and Rendleman and Bartter [18]. The main advantage of the method is the ease of implementation. The first binomial tree model for pricing Asian option was proposed by Hull and White [13] in 1993. Based on the binomial trees models, considerable research results have been reported on the Asian option pricing problem. For example, Klassen [19] proposed a modified binomial approach the smallest possible number of average values at each node based on the Hull and White model. Massimo, et al. [20] associated a set of representative averages chosen among all the effective averages realized at each node of the tree, and then use backward recursion and linear interpolation to compute the option price. Dai, et al. [21] proposed a more representative average prices in terms of the function of the arithmetic average price. Hsu and Lyuu [22] proposed a quadratic-time convergent binomial method based on the Lagrange multiplier to choose the number of states for each node of a tree. Kolkiewicz [23] proposed a method of hedging path-dependent options in a discrete-time setup under the Black-Scholes model.

In finance, most scholars usually model the stock prices directly, and most of them do not consider the dividend income from holding the stock, this practice contradicts with the fact that the stock pays dividends. Even if the payment of dividends is considered, the dividends are basically considered to be distributed continuously, which contradicts with the fact that dividends are distributed in discrete form. Arbitrage pricing theory (APT) tells us that the price of a stock in a company should be equal to the present value of the future dividend payments. From this point of view, it is more appropriate to model the dividend process according to the arbitrage pricing theory such that the stock price process becomes an evolutionary result of the dividend process.

Driffill, et al. [24] considered the American option pricing while the dividends 
process is a geometric Brownian motion model under regime switching. Korn and Rogers [25] discussed the problem of discrete dividend modeling and option pricing. Graziano and Rogers [26] assumed the dividends process to be a Markov-modulated geometric Brownian motion, and then computed the Basket option under the dividends model. Sakksa and Le [27] derived the corresponding dynamics of stock price and various option pricing formulae, while the discrete dividend processes incorporate the dependence of assets on the market mode and the state of the economy, where the latter is modeled by a hidden finite-state Markov chain. Shi and Liu [28] defined a looking back-reset option, and then gave the pricing formulas for this new option on the assets with constant dividend yield. Jeon, et al. [29] presented a study of American floating strike lookback options written on dividend-paying assets.

In the above references, both the dividend model and the binomial tree model have attracted considerable research interest, but few people combine these two models together to consider the pricing problem of discrete arithmetic average Asian option, moreover, the release of discrete dividends with binomial tree method has also not been investigated. Based on the above analysis, this paper considers the important impact of the market status, volatility with the dividend process and market risk. With the help of the arbitrage pricing theory and the dividend discount model, in this paper, a model with the Markov-modulated dividend process is proposed, and the binomial tree method is used to discuss the pricing of the discrete arithmetic average Asian option under the dividend model. Markov chain is employed to describe the changing rule of the stochastic volatility of the dividend model. Different from most of the existing results, this paper selects the binomial tree model which is from Rendleman and Bartter [18] not from Cox, et al. [17]. The reason for choosing this model is that $p$ is assumed to be equal to $\frac{1}{2}$ when we calculate the upper factor and lower factor. The upper factor and lower factor are also different from the general ones, our factors are changed in different intervals since the volatility of dividend process is a random variable. The variable factors make the price of the option more accurate.

The paper is divided into the following several parts: in Section 2, some preliminary theoretical knowledge including market models are given; in Section 3 , the stock price under the dividend model is given based on the arbitrage pricing theory; in Section 4, we compute the change percentage of the stock price under the original model and the binomial tree model, and the factors $u_{i}$ and $d_{i}$ are computed based on the change percentage, furthermore, the expression of the discrete arithmetic average Asian option is given under the binomial tree model; in Section 5, a simulation example is given to demonstrate the effectiveness of the proposed results; finally, conclusions are drawn in Section 6.

\section{Preliminary Knowledge}

In this paper, two assets has been considered in the market. One is the riskless asset (such as the bond) where the price $B(t)_{t \geq 0}$ satisfies 


$$
\mathrm{d} B(t)=B(t) r(t) \mathrm{d} t, \quad B(0)=1
$$

where $r(t)$ is the risk-free interest rate at time $t$. The other is the risky asset supposed to be the stock. $\left(\Omega, \mathcal{F},\left\{\mathcal{F}_{t}\right\}_{t \geq 0}, P\right)$ is a complete probability space where the filtration $\left\{\mathcal{F}_{t}\right\}_{t \geq 0}$ follows the usual conditions. The $[0, T]$ is the time interval, where 0 and $T$ represent the current date and the due date respectively. The stock pay dividend $D_{i}$ at discrete times $t_{i}$, where $t_{i}=i h$ ( $h$ is a fixed positive constant), and the dividend takes the form $D_{i}=\lambda X_{i}$ where $\lambda$ is a constant.

The discrete dividend $X(t)$ is assumed to follow the Geometric Brownian motion with Markov-modulated volatility:

$$
\frac{\mathrm{d} X_{t}}{X_{t}}=\mu \mathrm{d} t+\sigma\left(\xi_{t}\right) \mathrm{d} W(t)
$$

where $\sigma(\cdot)$ is the volatility of dividend process and $\xi_{t}$ is a Markov chain with finite-state $\{0,1,2, \cdots, k\}$, we assume the initial state to be $0, \sigma\left(\xi_{t_{0}}\right)=\xi(0)$ and $p_{i j}=p\left\{\xi_{t+\Delta t}=j \mid \xi_{t}=i\right\}$.

Here, we assume that the approached model working in the appropriate pricing measure. The APT implies that the stock price is given by the expected sum of all future dividends appropriately discounted, so that we have the following basic formula [25]

$$
S_{t}=E\left[\sum_{t_{m}>t} \beta\left(t_{m}\right) D_{m}\right] / \beta(t)
$$

where $\beta(t)=\exp \left(-\int_{0}^{t} r_{s} \mathrm{~d} s\right)$ is the discount factor with the interest rate $r_{s}$.

We assume that:

1) The announcement and payment times always coincide for the dividends.

2) The dividend process satisfies suitable growth conditions $(r>\mu)$ so that the above sum is always finite.

\section{The Stock Price under the Dividend Process}

If the dividend process $X(t)$ obeys the Geometric Brownian motion with Markov-modulated volatility, then we get

$$
X_{t}=X_{0} \exp \left\{\mu t-\int_{0}^{t} \frac{1}{2} \sigma\left(\xi_{s}\right)^{2} \mathrm{~d} s+\int_{0}^{t} \sigma\left(\xi_{s}\right) \mathrm{d} w_{s}\right\} .
$$

Taking the conditional expectation on the both sides of the above equation, we obtain:

$$
E\left[X_{t} \mid \mathcal{F}_{s}\right]=X_{s} \mathrm{e}^{(t-s)} .
$$

From(2.3), we can get the stock price [25] under the dividend process

$$
\begin{aligned}
S_{t} & =E\left[\sum_{t_{m}>t} \beta\left(t_{m}\right) D_{m}\right] / \beta(t)=\sum_{m \geq k} \mathrm{e}^{-r(m h-t)} \lambda E_{t} X_{m h} \\
& =\sum_{m \geq k} \mathrm{e}^{-r(m h-t)} \lambda X_{t} \mathrm{e}^{\mu(m h-t)}=\frac{\lambda X_{t} \mathrm{e}^{-(r-\mu)(k h-t)}}{1-\mathrm{e}^{-(r-\mu) h}}
\end{aligned}
$$


for $t \in[(k-1) h, k h)$, and

$$
\begin{aligned}
S_{t} & =E_{t}\left[\sum_{i>m} \beta\left(t_{i}\right) D_{i}\right] / \beta(t)=\sum_{i \geq m} \mathrm{e}^{-r(i h-t)} \lambda E_{t} X_{i h} \\
& =\sum_{i \geq m} \mathrm{e}^{-r(i h-t)} \lambda X_{t} \mathrm{e}^{\mu(i h-t)}-\lambda X_{t} \\
& =\frac{\lambda X_{t} \mathrm{e}^{-(r-\mu) h}}{1-\mathrm{e}^{-(r-\mu) h}}
\end{aligned}
$$

for $t=m h, S_{t}=S_{t-}-D_{m}, \quad S_{t-}=S_{t}+D_{m}=\frac{\lambda X_{t}}{1-\mathrm{e}^{-(r-\mu) h}}$.

\section{Binomial Tree Based Models for Pricing Asian Option}

In this section, we give new factors $u_{i}$ and $d_{i}$ instead of $u$ and $d$ proposed by Cox, Ross and Rubinstein [17]. Then the price of Asian option can be computed with the new parameters. Without losing generality, we only consider the case of no dividend payment or dividend payment once within the validity period of the option.

Firstly, we assume that:

1) Discretizing the time period $[0, T]$ into $n$ intervals of the same length $\Delta t=\frac{T}{N}, t_{i}=i \Delta t, \quad i=0,1,2, \cdots, N$.

2) The stock price $S_{t_{i}}$ at $t=i \Delta t$ moves up to $S_{t_{i+1}}=S_{t_{i}} u_{i}$ or down to $S_{t_{i+1}}=S_{t_{i}} d_{i}$ with probabilities $p$ or $1-p$, respectively.

3) The volatility $\sigma(\cdot)$ is not changed in each interval.

\subsection{Case One: No Dividend Payment within the Validity Period of the Option}

When the stock price $S_{t_{i-1}}$ moves to $S_{t_{i}}$ over the period $\left[t_{i-1}, t_{i}\right]$, the changing percentage of the stock price is denoted by $Y_{i}=\frac{S_{t_{i}}}{S_{t_{i-1}}}$. The first moment and the second moment of $Y_{i}$ in our original model can be given by:

$$
\begin{aligned}
E\left[Y_{i}\right] & =E\left[\frac{X_{t_{i}}}{X_{t_{i-1}}} \mathrm{e}^{(r-\mu) \Delta t}\right] \\
& =E\left[\frac{X_{0} \exp \left\{\mu i \Delta t-\int_{0}^{i \Delta t} \frac{1}{2} \sigma^{2}\left(\xi_{t}\right) \mathrm{d} s+\int_{0}^{i \Delta t} \sigma\left(\xi_{t}\right) \mathrm{d} w_{s}\right\}}{\left.X_{0} \exp \left\{\mu(i-1) \Delta t-\int_{0}^{(i-1) \Delta t} \frac{1}{2} \sigma^{2}\left(\xi_{t}\right) \mathrm{d} s+\int_{0}^{(i-1) \Delta t} \sigma\left(\xi_{t}\right) \mathrm{d} w_{s}\right\}\right] \mathrm{e}^{(r-\mu) \Delta t}}\right. \\
& =E\left[\exp \left\{\mu \Delta t-\int_{(i-1) \Delta t}^{i \Delta t} \frac{1}{2} \sigma^{2}\left(\xi_{t}\right) \mathrm{d} s+\int_{(i-1) \Delta t}^{i \Delta t} \sigma\left(\xi_{t}\right) \mathrm{d} w_{s}\right\}\right] \mathrm{e}^{(r-\mu) \Delta t} \\
& =E\left[\mathrm{e}^{\mu \Delta t}\right] E\left[\exp \left\{\int_{(i-1) \Delta t}^{i \Delta t}-\frac{1}{2} \sigma^{2}\left(\xi_{t}\right) \mathrm{d} s+\int_{(i-1) \Delta t}^{i \Delta t} \sigma\left(\xi_{t}\right) \mathrm{d} w_{s}\right\}\right] \mathrm{e}^{(r-\mu) \Delta t} \\
& =\mathrm{e}^{\mu \Delta t} \mathrm{e}^{(r-\mu) \Delta t}=\mathrm{e}^{r \Delta t},
\end{aligned}
$$




$$
\begin{aligned}
& E\left[Y_{i}\right]^{2}=E\left[\frac{X_{i \Delta t}}{X_{(i-1) \Delta t}} \mathrm{e}^{(r-\mu) \Delta t}\right]^{2} \\
& =E\left[\frac{X_{0}^{2} \exp \left\{2 \mu i \Delta t-\int_{0}^{i \Delta t} \sigma^{2}\left(\xi_{t}\right) \mathrm{d} s+\int_{0}^{i \Delta t} 2 \sigma\left(\xi_{t}\right) \mathrm{d} w_{s}\right\}}{X_{0}^{2} \exp \left\{2 \mu(i-1) \Delta t-\int_{0}^{(i-1) \Delta t} \sigma^{2}\left(\xi_{t}\right) \mathrm{d} s+\int_{0}^{(i-1) \Delta t} 2 \sigma\left(\xi_{t}\right) \mathrm{d} w_{s}\right\}}\right] \mathrm{e}^{2(r-\mu) \Delta t} \\
& =E\left[\exp \left\{2 \mu \Delta t-\int_{(i-1) \Delta t}^{i \Delta t} \sigma^{2}\left(\xi_{t}\right) \mathrm{d} s+\int_{(i-1) \Delta t}^{i \Delta t} 2 \sigma\left(\xi_{t}\right) \mathrm{d} w_{s}\right\}\right] \mathrm{e}^{2(r-\mu) \Delta t} \\
& =E\left[\exp \left\{2 \mu \Delta t-\int_{(i-1) \Delta t}^{i \Delta t} \sigma^{2}\left(\xi_{t}\right) \mathrm{d} s+\int_{(i-1) \Delta t}^{i \Delta t} 2 \sigma^{2}\left(\xi_{t}\right) \mathrm{d} s\right\}\right] \\
& =\left[\exp \left\{-\int_{(i-1) \Delta t}^{i \Delta t} 2 \sigma^{2}\left(\xi_{t}\right) \mathrm{d} s+\int_{(i-1) \Delta t}^{i \Delta t} 2 \sigma\left(\xi_{t}\right) \mathrm{d} w_{s}\right\}\right] \mathrm{e}^{2(r-\mu) \Delta t} \\
& =\mathrm{e}^{2 \mu \Delta t} E\left[\exp \left\{\int_{(i-1) \Delta t}^{i \Delta t} \sigma^{2}\left(\xi_{t}\right) \mathrm{d} s\right\}\right] \mathrm{e}^{2(r-\mu) \Delta t} \\
& =\mathrm{e}^{2 \mu \Delta t} \sum_{j=0}^{k} p_{0 j}(i-1) \mathrm{e}^{\sigma^{2}(j) \Delta t} \mathrm{e}^{2(r-\mu) \Delta t} \\
& =\mathrm{e}^{2 r \Delta t} \sum_{j=0}^{k} p_{0 j}(i-1) \mathrm{e}^{\sigma^{2}(j) \Delta t} .
\end{aligned}
$$

The first moment and the second moment of $Y_{i}$ in the binomial tree-based model could be written as follow:

$$
\begin{gathered}
E\left[Y_{i}\right]=p u_{i}+(1-p) d_{i}, \\
E\left[Y_{i}\right]^{2}=p u_{i}^{2}+(1-p) d_{i}^{2} .
\end{gathered}
$$

As the mean and the variance of $Y_{i}$ under the binomial tree-based model should be equal to that under the original model over the period $\left[t_{i-1}, t_{i}\right]$. We match them and set up the system of equations. Let $p=\frac{1}{2}$, according to Rendleman and Bartter [18], we have

$$
\left\{\begin{array}{l}
p u_{i}+(1-p) d_{i}=\mathrm{e}^{r \Delta t} \\
p u_{i}^{2}+(1-p) d_{i}^{2}=\mathrm{e}^{2 r \Delta t} \sum_{j=0}^{k} p_{0 j}(i-1) \mathrm{e}^{\sigma^{2}(j) \Delta t} \\
p=\frac{1}{2}
\end{array}\right.
$$

From(4.5), we have:

$$
\begin{aligned}
& u_{i}=\mathrm{e}^{r \Delta t}+\mathrm{e}^{r \Delta t} \sqrt{\sum_{j=0}^{k} p_{0 j}(i-1) \mathrm{e}^{\sigma^{2}(j) \Delta t}-1}, \\
& d_{i}=\mathrm{e}^{r \Delta t}-\mathrm{e}^{r \Delta t} \sqrt{\sum_{j=0}^{k} p_{0 j}(i-1) \mathrm{e}^{\sigma^{2}(j) \Delta t}-1 .}
\end{aligned}
$$

\subsection{Case Two: Once Dividend Payment within the Validity Period of the Option}

If the dividend payment occurs in the $m$-th interval, then the changing percentage 
of the stock price in this interval is $Y_{m}=\frac{X_{t_{m}}}{X_{t_{m-1}}} \mathrm{e}^{-(r-\mu)(h-\Delta t)}$. Furthermore, the first moment and second moment of $Y_{i}$ in our original model is given by:

$$
\begin{gathered}
E\left[Y_{m}\right]=E\left[\frac{X_{t_{m}}}{X_{t_{m-1}}} \mathrm{e}^{-(r-\mu)(h-\Delta t)}\right]=\mathrm{e}^{r \Delta t-(r-\mu) h}, \\
E\left[Y_{m}\right]^{2}=E\left[\frac{X_{t_{m}}}{X_{t_{m-1}}} \mathrm{e}^{-(r-\mu)(h-\Delta t)}\right]^{2}=\mathrm{e}^{2 r \Delta t-2(r-\mu) h} \sum_{j=0}^{k} p_{0 j}(m-1) \mathrm{e}^{\sigma^{2}(j) \Delta t} .
\end{gathered}
$$

As the same analyzation in Case One, one has

$$
\left\{\begin{array}{l}
p u_{m}+(1-p) d_{m}=e^{r \Delta t-(r-\mu) h}, \\
p u_{m}^{2}+(1-p) d_{m}^{2}=\mathrm{e}^{2 r \Delta t-2(r-\mu) h} \sum_{j=0}^{k} p_{0 j}(m-1) \mathrm{e}^{\sigma^{2}(j) \Delta t}, \\
p=\frac{1}{2} .
\end{array}\right.
$$

Then, we can obtain following solutions:

$$
\begin{aligned}
& u_{m}=\mathrm{e}^{r \Delta t-(r-\mu) h}+\mathrm{e}^{r \Delta t-(r-\mu) h} \sqrt{\sum_{j=0}^{k} p_{0 j}(m-1) \mathrm{e}^{\sigma^{2}(j) \Delta t}-1}, \\
& d_{m}=\mathrm{e}^{r \Delta t-(r-\mu) h}-\mathrm{e}^{r \Delta t-(r-\mu) h} \sqrt{\sum_{j=0}^{k} p_{0 j}(m-1) \mathrm{e}^{\sigma^{2}(j) \Delta t}-1} .
\end{aligned}
$$

Following the same analysis method, the factors in other period can be get by using the similar techniques in Case One and Two.

\subsection{Compute the Price of Asian Option}

The stock price path is shown in Figure 1 . When $t=t_{0}$, the stock prices and the sum of the price are set as $S_{0}^{1}=S_{0}$ and $M_{0}^{1}=S_{0}$, respectively. Then, at time $t_{1}$ the stock prices on the node of each path are $S_{1}^{1}=S_{0}^{1} u_{1}, S_{1}^{2}=S_{0}^{1} d_{1}$ and the sum of the prices on the node of each path are $M_{1}^{1}=M_{0}^{1}+S_{1}^{1}, M_{1}^{2}=M_{0}^{1}+S_{1}^{2}$ from top to bottom. The probability that the stock price passes through each path is

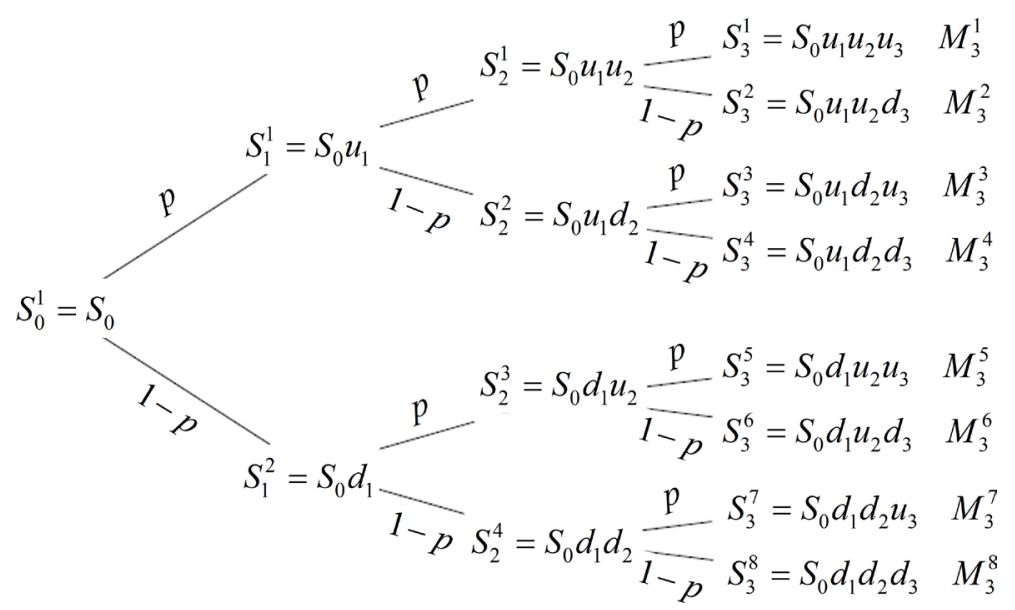

Figure 1. Stock price path. 
$\frac{1}{2}$. At time $t_{2}$, the stock prices on the node of each path are $S_{2}^{1}=S_{1}^{1} u_{2}$, $S_{2}^{2}=S_{1}^{1} d_{2}, S_{2}^{3}=S_{1}^{2} u_{2}, S_{2}^{4}=S_{1}^{2} d_{2}$ and the sum of the prices on the node of each path are $M_{2}^{1}=M_{1}^{1}+S_{2}^{1}, \quad M_{2}^{2}=M_{1}^{1}+S_{2}^{2}, \quad M_{2}^{3}=M_{1}^{2}+S_{2}^{3}, \quad M_{2}^{4}=M_{1}^{2}+S_{2}^{4}$ from top to bottom. The probability that the stock price passes through each path is $\frac{1}{4}$. In general, at time $t_{i}$, the stock prices on the node of each path are $S_{i}^{j}=S_{i-1}^{\frac{j+1}{2}} u_{i}$ when $j$ is an odd number, and $S_{i}^{j}=S_{i-1}^{\frac{j}{2}} d_{i}$ when $j$ is an even number. The sum of the prices on the node of each path are $M_{i}^{j}=M_{i-1}^{\frac{j+1}{2}}+M_{i}^{j}$ when $j$ is an odd number, and $M_{i}^{j}=M_{i-1}^{\frac{j}{2}}+M_{i}^{j}$ when $j$ is an even number from top to bottom. The probability that the stock price passes through each path is $\frac{1}{2^{i}}$.

Thus, the price of Asian option can be computed as follows:

$$
V\left(X_{0}, K, T, 0\right)=\mathrm{e}^{-r T} \frac{1}{2^{N}} \sum_{j=1}^{2^{N}} \max \left(\frac{M_{N}^{j}}{N+1}-K, 0\right) .
$$

\section{Numeral Calculations}

In this section, we shall present the numerical results to demonstrate the effectiveness of the proposed model. In order to simplify the simulation, we assume that the chain $\xi_{t}$ has only two states $e_{0}$ and $e_{1}$ to express prosperity and depression of economy respectively, the initial state $\xi_{t_{0}}=e_{0}$. In these states, the values of the parameters are given as $\sigma\left(e_{0}\right)=0.1, \sigma\left(e_{1}\right)=0.3$. The transfer probability matrix is selected as

$$
P=\left[\begin{array}{ll}
0.7 & 0.3 \\
0.2 & 0.8
\end{array}\right]
$$

$X_{0}=0.38, r=0.03, \mu=0.02, \Delta t=\frac{1}{12}$. We only consider the case of once dividend payment within the validity period of the option.

When the option expiry time takes from $\frac{1}{12}$ to $\frac{15}{12}$, the price of the discrete arithmetic average Asian option is shown in Table 1 for different strike prices and different times of the dividend payment. From the table, we can see addition of the price of the discrete arithmetic average Asian option as the expiration time increases. The price of the option is not only related to the expiry date, but also to the strike price and the time of dividend payment. In order to show the impact of these factors on the option price better, we give the following figure.

In Figures 2-4, we can see that the dividend payment will reduce the price of the option, and the sooner the dividend payment, when the expiry time are same, the greater the impact on the option. For example, if the expiry time is fixed as one year, it could be seen form Figure 3 that the price of the option when the dividend pay in the third interval is cheaper than the one when the dividend pay 
Table 1. Price of the discrete arithmetic average Asian option.

\begin{tabular}{|c|c|c|c|c|c|c|c|}
\hline \multicolumn{4}{|c|}{ Dividend pay in the third interval } & \multicolumn{4}{|c|}{ Dividend pay in the sixth interval } \\
\hline $\mathrm{N}$ & $\mathrm{K}=45$ & $\mathrm{~K}=50$ & $\mathrm{~K}=55$ & $\mathrm{~N}$ & $\mathrm{~K}=45$ & $\mathrm{~K}=50$ & $\mathrm{~K}=55$ \\
\hline 1 & 5.3372 & 0.5378 & 0 & 1 & 5.3372 & 0.5378 & 0 \\
\hline 2 & 5.3866 & 0.7673 & 0 & 2 & 5.3866 & 0.7673 & 0 \\
\hline 3 & 5.2487 & 0.9371 & 0 & 3 & 5.4358 & 1.0375 & 0 \\
\hline 4 & 5.1858 & 1.1085 & 0.0094 & 4 & 5.4849 & 1.2873 & 0.0316 \\
\hline 5 & 5.1772 & 1.2884 & 0.0751 & 5 & 5.5412 & 1.5000 & 0.1031 \\
\hline 6 & 5.2081 & 1.4826 & 0.1429 & 6 & 5.5151 & 1.6578 & 0.1798 \\
\hline 7 & 5.2629 & 1.6580 & 0.2374 & 7 & 5.5249 & 1.8100 & 0.2774 \\
\hline 8 & 5.3388 & 1.8275 & 0.3349 & 8 & 5.5655 & 1.9640 & 0.3788 \\
\hline 9 & 5.4220 & 1.9951 & 0.4436 & 9 & 5.6212 & 2.1164 & 0.4864 \\
\hline 10 & 5.5142 & 2.1543 & 0.5551 & 10 & 5.6904 & 2.2644 & 0.5964 \\
\hline 11 & 5.6098 & 2.3091 & 0.6659 & 11 & 5.7688 & 2.4086 & 0.7077 \\
\hline 12 & 5.7079 & 2.4580 & 0.7790 & 12 & 5.8516 & 2.5498 & 0.8194 \\
\hline 13 & 5.8073 & 2.6022 & 0.8916 & 13 & 5.9383 & 2.6867 & 0.931 \\
\hline 14 & 5.9071 & 2.7419 & 1.0038 & 14 & 6.0275 & 2.8203 & 1.0421 \\
\hline 15 & 6.0067 & 2.8773 & 1.1154 & 15 & 6.1178 & 2.9506 & 1.1525 \\
\hline \multicolumn{4}{|c|}{ Dividend pay in the ninth interval } & \multicolumn{4}{|c|}{ Dividend pay in the twelfth interval } \\
\hline $\mathrm{N}$ & $\mathrm{K}=45$ & $\mathrm{~K}=50$ & $\mathrm{~K}=55$ & $\mathrm{~N}$ & $\mathrm{~K}=45$ & $\mathrm{~K}=50$ & $\mathrm{~K}=55$ \\
\hline 1 & 5.3372 & 0.5378 & 0 & 1 & 5.3372 & 0.5378 & 0 \\
\hline 2 & 5.3866 & 0.7673 & 0 & 2 & 5.3866 & 0.7673 & 0 \\
\hline 3 & 5.4358 & 1.0375 & 0 & 3 & 5.4358 & 1.0375 & 0 \\
\hline 4 & 5.4849 & 1.2873 & 0.0316 & 4 & 5.4849 & 1.2873 & 0.0316 \\
\hline 5 & 5.5412 & 1.5000 & 0.1031 & 5 & 5.5412 & 1.5000 & 0.1031 \\
\hline 6 & 5.6182 & 1.7209 & 0.1953 & 6 & 5.6182 & 1.7209 & 0.1953 \\
\hline 7 & 5.7019 & 1.9220 & 0.3119 & 7 & 5.7019 & 1.9220 & 0.3119 \\
\hline 8 & 5.7964 & 2.1123 & 0.4297 & 8 & 5.7964 & 2.1123 & 0.4297 \\
\hline 9 & 5.8258 & 2.2480 & 0.5371 & 9 & 5.8947 & 2.2940 & 0.5551 \\
\hline 10 & 5.8728 & 2.3831 & 0.6452 & 10 & 5.9964 & 2.4652 & 0.6810 \\
\hline 11 & 5.9323 & 2.5166 & 0.7551 & 11 & 6.0995 & 2.6303 & 0.8073 \\
\hline 12 & 6.0000 & 2.6484 & 0.8657 & 12 & 6.1521 & 2.7521 & 0.9161 \\
\hline 13 & 6.0740 & 2.7777 & 0.9755 & 13 & 6.2129 & 2.8737 & 1.0242 \\
\hline 14 & 6.1520 & 2.9047 & 1.0851 & 14 & 6.2799 & 2.9938 & 1.1318 \\
\hline 15 & 6.2330 & 3.0292 & 1.1938 & 15 & 6.3512 & 3.1121 & 1.2389 \\
\hline \multicolumn{4}{|c|}{ Dividend pay in the fifteenth interval } & \multicolumn{4}{|c|}{ No dividend payment } \\
\hline $\mathrm{N}$ & $\mathrm{K}=45$ & $\mathrm{~K}=50$ & $\mathrm{~K}=55$ & $\mathrm{~N}$ & $\mathrm{~K}=45$ & $\mathrm{~K}=50$ & $\mathrm{~K}=55$ \\
\hline 1 & 5.3372 & 0.5378 & 0 & 1 & 5.3372 & 0.5378 & 0 \\
\hline 2 & 5.3866 & 0.7673 & 0 & 2 & 5.3866 & 0.7673 & 0 \\
\hline 3 & 5.4358 & 1.0375 & 0 & 3 & 5.4358 & 1.0375 & 0 \\
\hline 4 & 5.4849 & 1.2873 & 0.0316 & 4 & 5.4849 & 1.2873 & 0.0316 \\
\hline 5 & 5.5412 & 1.5000 & 0.1031 & 5 & 5.5412 & 1.5000 & 0.1031 \\
\hline 6 & 5.6182 & 1.7209 & 0.1953 & 6 & 5.6182 & 1.7209 & 0.1953 \\
\hline 7 & 5.7019 & 1.9220 & 0.3119 & 7 & 5.7019 & 1.9220 & 0.3119 \\
\hline 8 & 5.7964 & 2.1123 & 0.4297 & 8 & 5.7964 & 2.1123 & 0.4297 \\
\hline 9 & 5.8947 & 2.2940 & 0.5551 & 9 & 5.8947 & 2.2940 & 0.5551 \\
\hline 10 & 5.9964 & 2.4652 & 0.681 & 10 & 5.9964 & 2.4652 & 0.6810 \\
\hline 11 & 6.0995 & 2.6303 & 0.8073 & 11 & 6.0995 & 2.6303 & 0.8073 \\
\hline 12 & 6.2035 & 2.7876 & 0.9336 & 12 & 6.2035 & 2.7876 & 0.9336 \\
\hline 13 & 6.3069 & 2.9396 & 1.0584 & 13 & 6.3069 & 2.9396 & 1.0584 \\
\hline 14 & 6.4102 & 3.0859 & 1.1818 & 14 & 6.4102 & 3.0859 & 1.1818 \\
\hline 15 & 6.4718 & 3.1982 & 1.2869 & 15 & 6.5124 & 3.2274 & 1.3034 \\
\hline
\end{tabular}




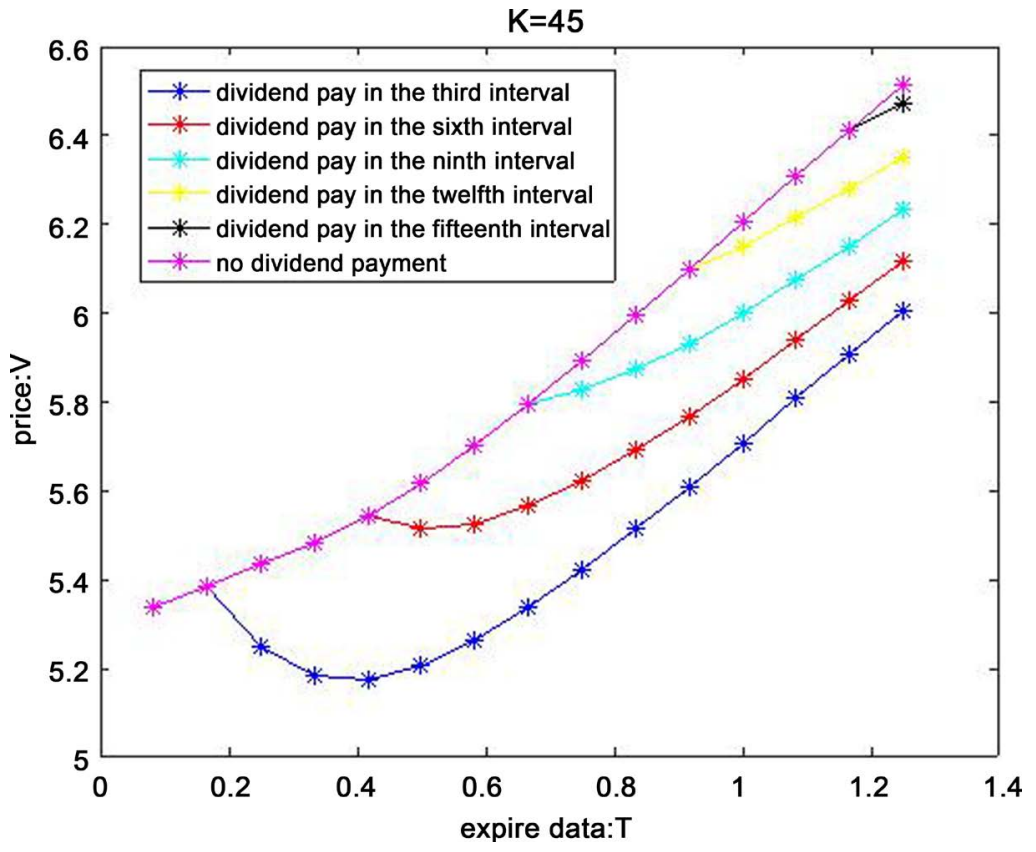

Figure 2. The strike price $K=45$.

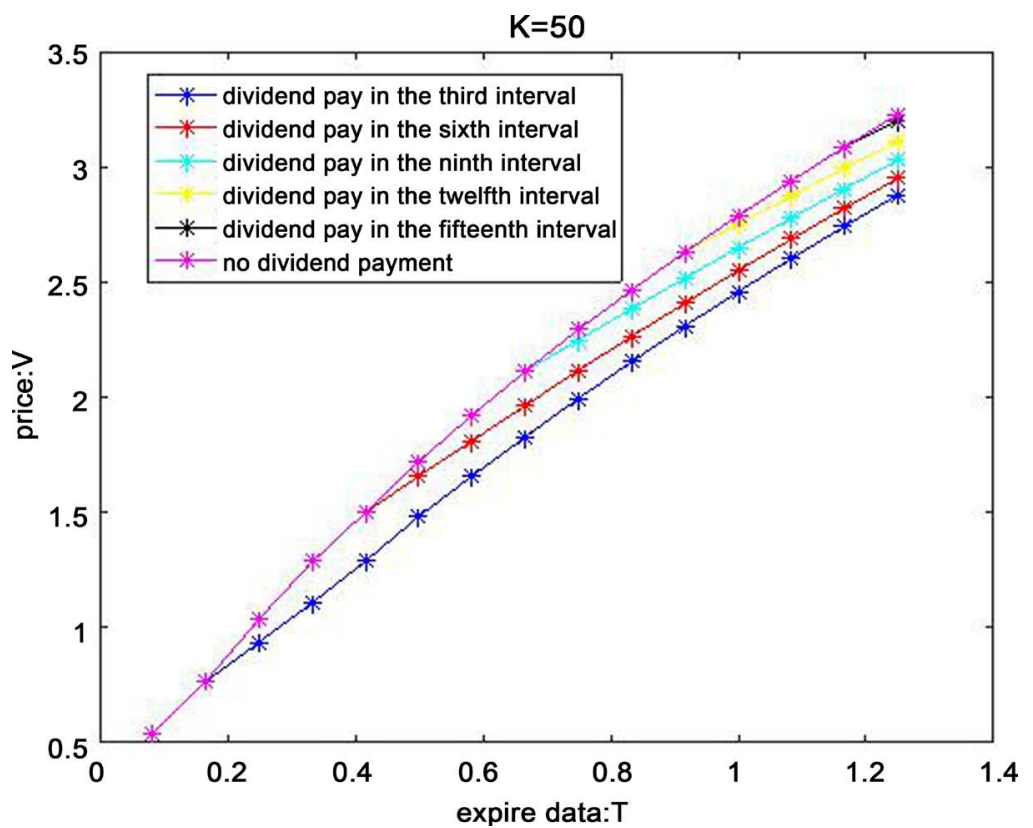

Figure 3. The strike price $K=50$.

in the ninth interval. Form Figure 2, we can see the impact of the dividend payment on the option price is significant when the strike price is 45 . And we can also get the information that option dropped a lot suddenly if the interval between the start time and expiration time is small and the dividend payment between the interval.

The image of the above figures is consistent with the financial markets. The value of the option is reflected in two aspects, one is the intrinsic value and the other is the time value. The intrinsic value of the call option is equal to the stock 


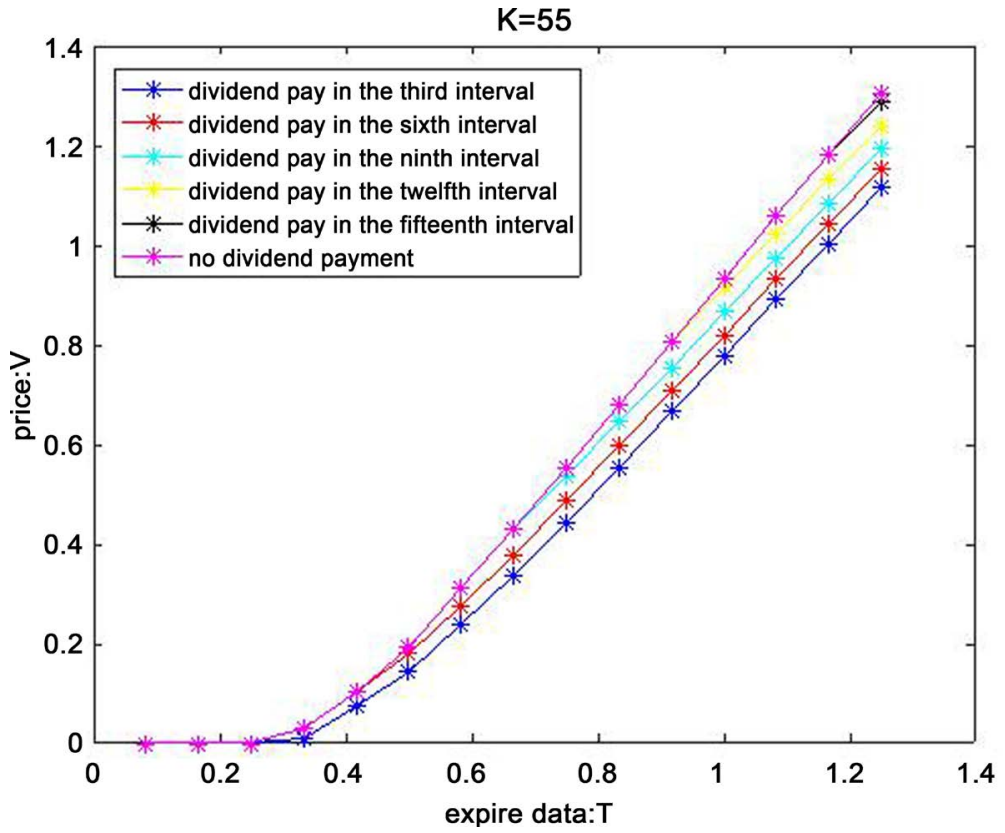

Figure 4. The strike price $K=55$.

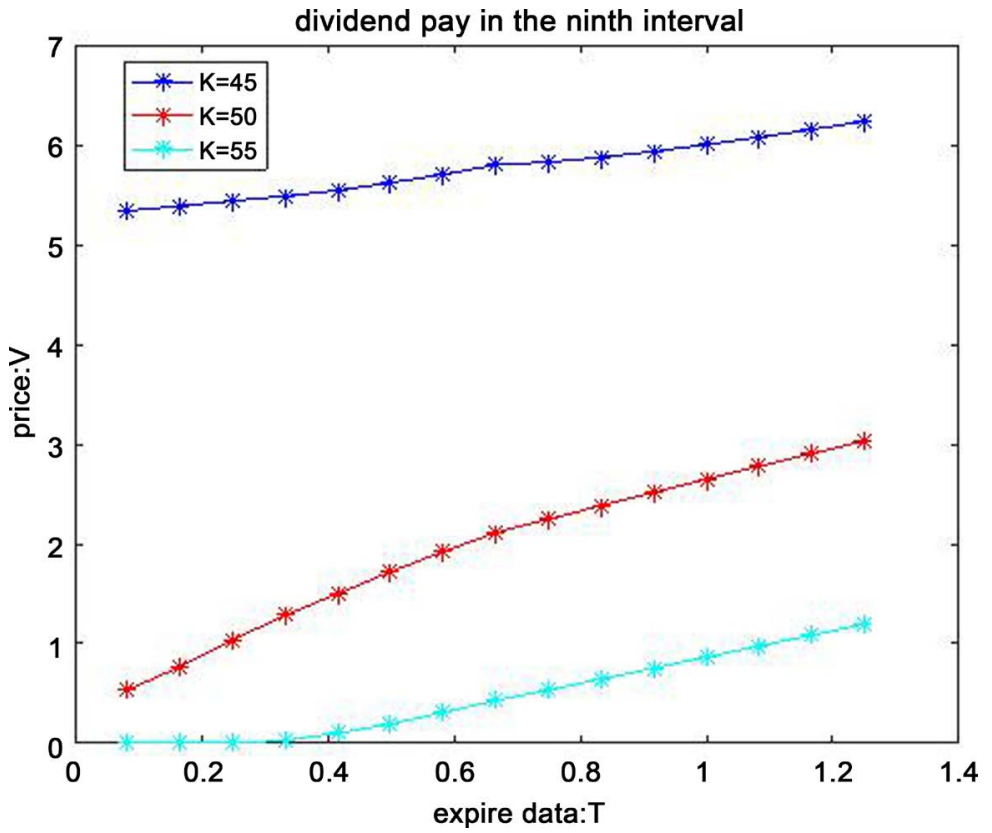

Figure 5. Dividend pay in the ninth interval.

price minus the outstanding value of the option. Obviously, the dividend led to the reduction of the intrinsic value. The value of the stock is the discount to all future cash flows, dividends are nothing more than a part of the current value of cash. For example, the ten dollars stock pay one dollar dividend, resulting in a lower stock price, stock price drops to nine dollars naturally. So when the value of time does not change, the payment of the dividend leads to the decrease in the intrinsic value of the option, which further results in the decrease in the option price.

In Figure 5, we can see that the option price is higher if the strike price is 
lower for other fixed influencing factors. This is because when the strike price is lower the possibility of the execution is bigger, and thus the option price is higher. Conversely, the option price will be lower, but will not be negative. The change of strike price is also changing the intrinsic value of the option.

In addition, both in above figures, we can see that the price of options is getting higher and higher as the expiry time increasing. This is because the expiry time changes the time premium of the option.

\section{Conclusion}

In this paper, the binomial tree method is used to calculate the price of the discrete arithmetic average Asian option, and the binomial tree model is determined by calculating the upper and lower factors. Finally, the validity of the method is verified by numerical calculation. However, the method is not feasible when the segmentation interval is relatively large.

\section{Fund}

This work was supported in part by the National Nature Science Foundations of China under Grant No. 61673103 and No. 61403248.

\section{References}

[1] Hull, J. and White, A. (1987) The Pricing of Options on Assets with Stochastic Volatilities. The Journal of Finance, 42, 281-300.

[2] Deng, G.H. (2015) Pricing American Continuous-Installment Options under Stochastic Volatility Model. Journal of Mathematical Analysis and Applications, 424, 802-823. https://doi.org/10.1016/j.jmaa.2014.11.049

[3] Merton, R.C. (1976) Option Pricing When Underlying Stock Return Are Discontinuous. Journal of Economics, 3, 125-144.

[4] Vecer, J. (2014) Black-Scholes Representation for Asian Options. Mathematical Finance, 24, 598-626.

[5] Benth, B.S. and Detering, N. (2015) Pricing and Hedging Asian-Style Options on Energy. Finance and Stochastics, 19, 849-889. https://doi.org/10.1007/s00780-015-0270-2

[6] Wang, H.E. and Li, K.D. (2007) Actuarial Pricing of Asian Option. Huazhong University of Science and Technology, Wuhan. (In Chinese)

[7] Kim, S. (2009) On a Degenerate Parabolic Equation Arising in Pricing of Asian Options. Journal of Mathematical Analysis and Applications, 351, 326-333. https://doi.org/10.1016/j.jmaa.2008.10.019

[8] Dai, T.S. and Chiu, C.Y. (2014) Pricing Barrier Stock Options with Discrete Dividends by Approximating Analytical Formulae. Quantitative Finance, 14, 1367-1382. https://doi.org/10.1080/14697688.2013.853319

[9] Juan, L. (2017) Pricing Power Options with Jump-Diffusion Markov-Modulated. Donghua University, Shanghai. (In Chinese)

[10] Yan, H.H., Shu, H.S. and Kan, X. (2015) Pricing Equity-Indexed Annuities When Discrete Dividends Follow a Markov-Modulated Jump Diffusion Model. Communications in Statistics-Theory and Methods, 44, 2207-2221. 
https://doi.org/10.1080/03610926.2013.819922

[11] Lian, Y.Y. and Zhang, T. (2010) Construction of New Binomial Tree Parameter Model for Option Pricing. The Practice and Understanding of Mathematics. (In Chinese)

[12] Kemn, A. and Vorst, A. (1990) A Pricing Method for Options Based on Average Asset Price. Journal of Banking and Finance, 2, 52-66.

[13] Hull, J.C. and White, A.D. (1993) Efficient Procedures for Valuing European and American Path-Dependent Options. The Journal of Derivatives, 1, 21-31. https://doi.org/10.3905/jod.1993.407869

[14] Rogers, L. and Shi, Z. (1995) The Value of an Asian Option. Journal of Applied Probability, 32, 1077-1088. https://doi.org/10.1017/S0021900200103559

[15] Hishida, Y. and Yasutomi, K. (2006) On the Asymptotic Behavior of the Prices of Asian Options. Asia-Pacific Financial Markets, 12, 289-306. https://doi.org/10.1007/s10690-006-9027-4

[16] Boyle, P. and Potapchik, A. (2008) Prices and Sensitivities of Asian Options: A Survey. Insurance: Mathematics and Economics, 42, 189-211. https://doi.org/10.1016/j.insmatheco.2007.02.003

[17] Cox, J.C., Ross, A. and Rubinstenin, M. (1979) Option Pricing: A Simplified Approach. Journal of Financial Economics, 7, 229-263. https://doi.org/10.1016/0304-405X(79)90015-1

[18] Rendleman, R.J. and Bartter, B.J. (1979) Two-State Option Pricing. The Journal of Finance, 34, 1093-1110.

[19] Klassen, T.R. (2001) Simple, Fast and Flexible Pricing of Asian Options. Journal of Computational Finance, 4, 89-124. https://doi.org/10.21314/JCF.2001.067

[20] Massimo, C., Ivar, M. and Emilio, R. (2006) An Adjusted Binomial Model for Pricing Asian Options. Review of Quantitative Finance and Accounting, 27, 258-296.

[21] Dai, T.S., Wang, J.Y. and Wei, H.S. (2008) Adaptive Placement Method on Pricing Arithmetic Average Options. Review of Derivatives Researches, 11, 83-118. https://doi.org/10.1007/s11147-008-9025-y

[22] Hsu, W.Y. and Lyuu, Y.D. (2011) Efficient Pricing of Discrete Asian Options. Applied Mathematics and Computation, 217, 9875-9894. https://doi.org/10.1016/j.amc.2011.01.015

[23] Kolkiewicz, A.W. (2016) Efficient Hedging of Path-Dependent Options. International Journal of Theoretical and Applied Finance, 19, Article ID: 1650032. https://doi.org/10.1142/S0219024916500321

[24] Driffill, J., Kenc, T. and Sola, M. (2002) Merton-Style Option Pricing under Regime Switching. Computing in Economics and Finance, 1-33.

[25] Korn, R. and Rogers, L. (2005) Stocks Paying Discrete Dividends: Modeling and Option Pricing. Journal of Derivatives, 13, 44-48. https://doi.org/10.3905/jod.2005.605354

[26] Di Graziano, G. and Rogers, L. (2006) Barrier Option Pricing for Assets with Markov-Modulated Dividends. Journal of Computational Finance, 9, 75-87. https://doi.org/10.21314/JCF.2006.151

[27] Sakksa, E. and Le, H. (2009) A Markov-Modulated Model for Stocks Paying Discrete Dividends. Insurance: Mathematics and Economics, 45, 19-24. https://doi.org/10.1016/j.insmatheco.2009.02.005

[28] Shi, W. and Liu, L. (2016) Pricing of the Looking Back-Reset Option with Barrier. 
Journal of Hebei University (Natural Science Edition), 40, 106-11.

[29] Jeon, J., Han, H. and Kang, M. (2017) Valuing American Floating Strike Lookback Option and Neumann Problem for Inhomogeneous Black-Scholes Equation. Journal of Computational and Applied Mathematics, 313, 218-234.

https://doi.org/10.1016/j.cam.2016.09.020 